\title{
Direct Imaging of Transient Interference in a Single-Mode Waveguide Using Near-Field Scanning Optical Microscopy
}

\author{
Guang Wei Yuan, Matthew D. Stephens, David S. Dandy, and Kevin L. Lear, Member, IEEE
}

\begin{abstract}
Near-field scanning optical microscopy was used to image transient interference between the guided mode and a leaky mode induced in a single-mode waveguide due to a localized adlayer. The observed field response in the adlayer region as well as the period and decay length of the subsequent interference are in good agreement with beam propagation calculations. The transient interference impacts the element spacing in local evanescent array coupled sensors.
\end{abstract}

Index Terms-Biosensor, evanescent field, leaky mode, near-field scanning optical microscopy (NSOM), optical interference, waveguide.

\section{INTRODUCTION}

A LOCAL, evanescent, array-coupled (LEAC) sensor based on a compact, single-mode optical waveguide is being developed for multianalyte sensing [1]. The LEAC sensor mechanism relies on specific binding of analytes to one of several localized regions of immobilized biological molecule probes, to modify the waveguide cross section and, thus, the local evanescent field. An array of detector elements along the length of the waveguide, each opposite a region of specific antibody type, can sense the modification in the evanescent field due to local adlayers of bound antigens.

To sense multiple adlayers at different positions along the waveguide, the optical field disturbance from one sensor element needs to be minimized or at least controlled before light is incident on the next one. However, each adlayer perturbs the local mode structure, thus exciting leaky modes in addition to the single bound mode. Transient optical interference or mode-beating between the bound and leaky modes, which have different propagation constants, causes subsequent oscillations in the evanescent field that damp as the leaky mode field dissipates. This letter reports for the first time both the experimental observation of this phenomenon with near-field scanning optical microscopy (NSOM) as well as beam propagation method (BPM) simulations that are in good agreement with the NSOM results.

Manuscript received June 15, 2005; revised August 5, 2005. This work was supported by the National Institutes of Health (NIH) under Grant EB00726.

G. W. Yuan and K. L. Lear are with Department of Electrical and Computer Engineering, Colorado State University, Fort Collins, CO 80523 USA (e-mail: gwyuan@engr.colostate.edu; kllear@engr.colostate.edu).

M. D. Stephens and D. S. Dandy are with Department of Chemical Engineering, Colorado State University, Fort Collins, CO 80523 USA (e-mail: stephmd@engr.colostate.edu; dandy@ colostate.edu).

Digital Object Identifier 10.1109/LPT.2005.858151

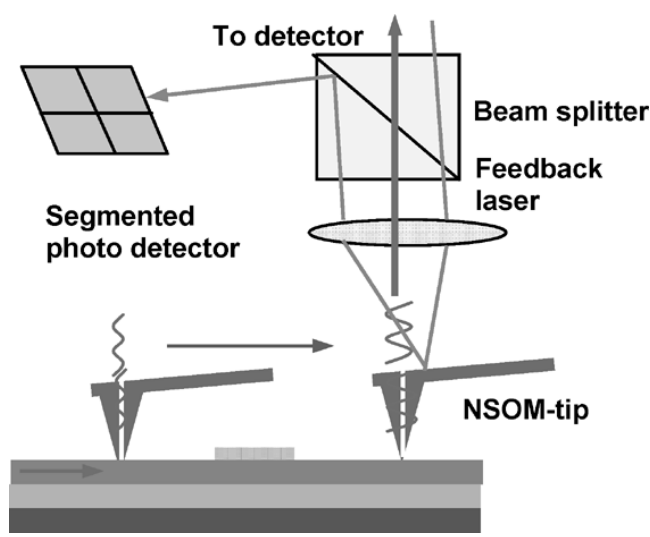

Fig. 1. Schematic diagram of the NSOM system for imaging the optical distribution in a waveguide.

\section{EXPERIMENTAL APPARATUS}

To analyze the interference phenomenon in a proof-of-concept configuration without the underlying detector array, NSOM is used to directly measure the evanescent field on the upper surface of the waveguide. The NSOM technique has been frequently used for optical waveguide characterization in light propagation properties, spatial modes profile, and guided modes interference [2]-[5]. NSOM measurements reported here employed an Alpha-NSOM system from WiTec with simultaneous optical and topological profiling capability. The experimental setup is shown in Fig. 1. The combination atomic force microscopy (AFM) and NSOM tip is a hollow aluminum pyramid integrated on a $\mathrm{Si}$ cantilever that converts the evanescent field to a propagating wave by locally frustrated total internal reflection [6]. A photomultiplier tube is aligned to a subwavelength diameter pinhole centered in the tip to detect the wave emanating from the tip that is proportional to the evanescent field. A reference laser beam reflecting off the cantilever is directed to a quadrant detector by a dichroic beam splitter and is used to sense tip deflection in AFM mode. As the tip is moved toward the top surface of the waveguide studied here, an exponentially increasing optical field is observed in addition to a constant signal due to reference beam scattering, verifying that the evanescent field can be distinguished from scattered light. During in-plane optical profiling, the NSOM was operated in contact mode so that the field at the top surface of the waveguide was measured.

The $\mathrm{SiN}_{x}-\mathrm{SiO}_{2}$ waveguide studied was fabricated using traditional sputtering, optical lithography, and etching methods. 

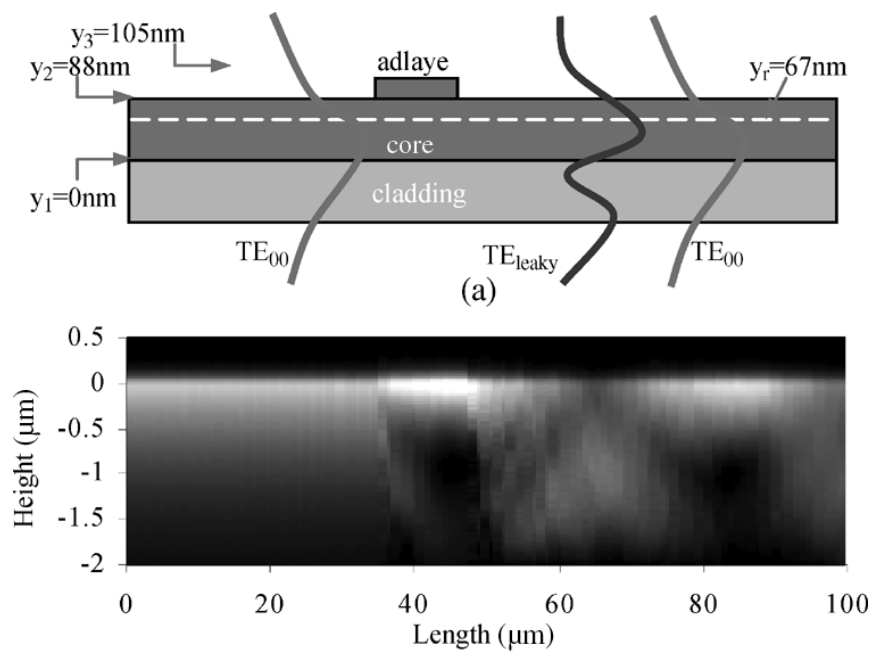

(b)

Fig. 2. (a) Cross section of waveguide and adlayer and (b) BPM simulation of transverse electric field magnitude.

A 105-nm-thick $\mathrm{SiN}_{x}$ film was deposited on a $\mathrm{SiO}_{2}-\mathrm{Si}$ wafer using $\mathrm{NH}_{3}$-argon sputtering, where the $\mathrm{SiO}_{2}$ cladding thickness is about $2 \mu \mathrm{m}$. Air serves as the upper cladding, allowing the NSOM to interrogate the evanescent field there. A 2- $\mu \mathrm{m}$-wide ridge waveguide core was defined by partially dry etching the surrounding $\mathrm{SiN}_{x}$ layer in a $\mathrm{CF}_{4}-\mathrm{O}_{2}$ plasma. The AFM determined the etch depth and, thus, ridge height to be $21 \mathrm{~nm}$. An artificial adlayer was created by masking $10-\mu \mathrm{m}$-wide bars oriented perpendicular to the waveguide, and etching the exposed $\mathrm{SiN}_{x}$ an additional $17 \mathrm{~nm}$. The waveguide core thickness after the adlayer etch is $88 \mathrm{~nm}$, and the ridge depth is $21 \mathrm{~nm}$. A longitudinal cross section of the resulting waveguide and adlayer structure is illustrated in Fig. 2(a) and a false intensity plot of the topology appears in Fig. 3. The three regions of the waveguide before, coincident with, and after the adlayer are referred to as Regions I, II, and III, respectively. Refractive indexes of the core and lower cladding are 1.8 and 1.45, respectively. Modal calculations determined that the waveguide supports only one guided mode $\mathrm{TE}_{00}$ in all three regions. Edge facets of the samples were polished for end-fire coupling of a 654-nm laser diode via visible single-mode fiber ( $4 / 125-\mu \mathrm{m}$ core-cladding diameter). Region II is located $4 \mathrm{~mm}$ from the input facet to assure a stable mode in Region I.

\section{Simulation AND MeAsurements}

Prior to performing NSOM experiments, optical propagation through the waveguide with adlayer structure was simulated by two-dimensional bidirectional BPM [7] using commercial software by RSoft. The incident beam was assumed to be the $\mathrm{TE}_{00}$ mode at a wavelength of the $654 \mathrm{~nm}$. The image of electric field magnitude in Fig. 2(b) clearly shows an interference effect in Region III. The interference is due to the excitation of a leaky mode in addition to the guided mode. Since the two modes have different propagation constants, the phase difference between them accumulates as they propagate, causing a sinusoidal intensity evolution due to mode beating. The effective indexes of the guided and dominant leaky mode in Region III are 1.459
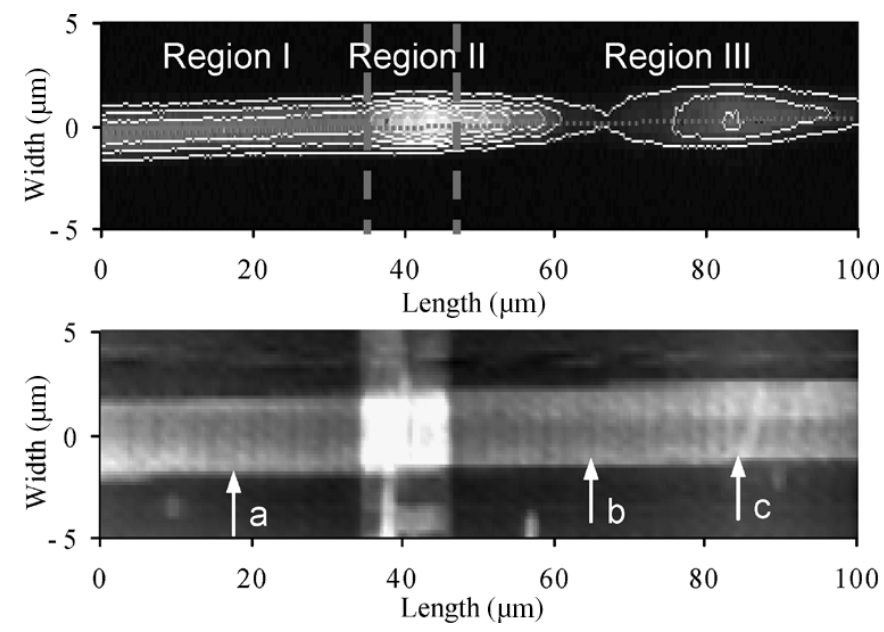

Fig. 3. (Top) Grayscale and contour plot of measured light intensity along the waveguide and (bottom) grayscale plot of the topography for the same region. The dotted line in the top portion shows the center line used for the longitudinal intensity plot in Fig. 5, and the arrows on the bottom portion show the positions for the transverse intensity plots in Fig. 4. Note that $x$ and $z$ scales are different.
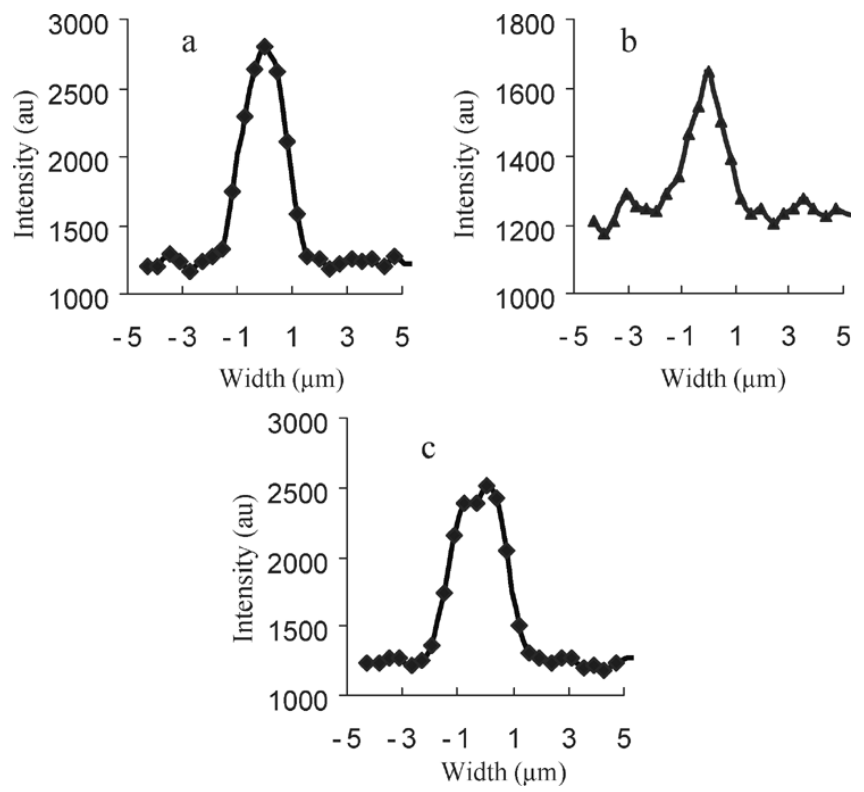

Fig. 4. Measured optical intensity distributions as a function of transverse position in the waveguide at positions along the waveguide indicated by the arrows in Fig. 3.

and 1.441 , respectively, so the leaky mode can propagate into the lower $\mathrm{SiO}_{2}$ (1.45 refractive index) cladding and gradually dissipate.

NSOM experiments yielded light intensity and topography maps simultaneously. Fig. 3(a) illustrates a contour plot of the measured light intensity over a $10 \times 100 \mu \mathrm{m}^{2}$ scanned area on the sample surface. Fig. 3(b) shows a simultaneously obtained topography image based on surface height information from the same scanned area. The waveguide width appears slightly larger than $2 \mu \mathrm{m}$ because of the NSOM tip size. The images clearly show a strong intensity change in the adlayer region and subsequent oscillations. Lateral optical field profiles are plotted in Fig. 4 at position (a) in Region I and positions (b) and (c) in Region III, as marked by arrows in Fig. 3 . 


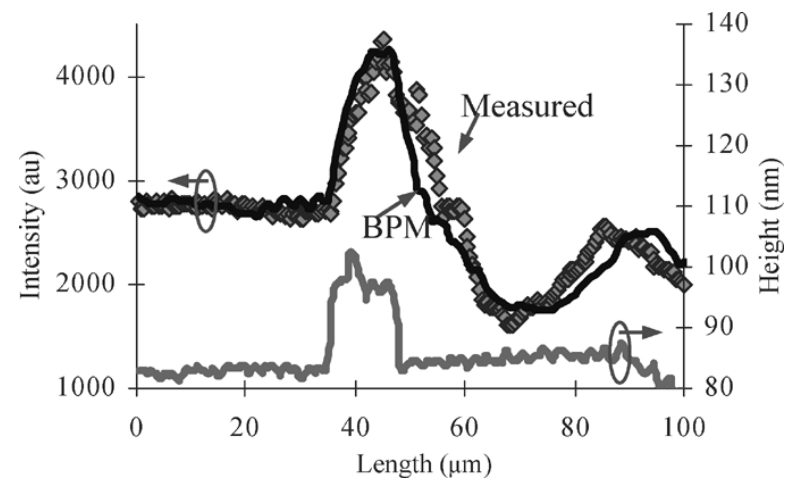

Fig. 5. Longitudinal dependence of the optical field (top) based on BPM simulation (solid line) and NSOM measured data (diamonds) and surface height (bottom).

The background intensity level is due to the scattered light from the reference laser and is constant at different positions. In position (a), before the adlayer, a Gaussian-shaped optical field profile corresponds to the guided $\mathrm{TE}_{00}$ mode in the waveguide. At positions (b) and (c) after the adlayer, the optical profiles represent destructive and constructive interference, respectively, between the guided mode and the leaky mode. Although the transverse profiles in Region III remain symmetric, their distributions are narrowed and broadened at points of destructive and constructive interference, respectively.

The intensity distribution along the center line of the waveguide from both NSOM measurements and BPM simulations are presented in Fig. 5, along with the surface height. The BPM simulation results, which include no adjustable parameters, agree well with the measured results. The damping of the oscillation was studied by shifting the $10 \times 100 \mu \mathrm{m}^{2}$ scanned area to fall completely in Region III. To improve the signal, a higher optical power was coupled into the waveguide, and the background due to the monitor laser scatter was subtracted from the data. An exponentially decaying intensity oscillation was observed, as shown in Fig. 6. Since leaky modes attenuate much faster than the guided mode, the decay rate can be attributed to the leaky mode attenuation. Fitting the measured data with an exponential decay curve produces an estimated attenuation coefficient of $152 \mathrm{~dB} / \mathrm{cm}$ for the leaky mode while the guided mode attenuation is $8 \mathrm{~dB} / \mathrm{cm}$. The average interference period is measured to be $36 \mu \mathrm{m}$, again in good agreement with the $38-\mu \mathrm{m}$ period predicted by BPM simulations and corresponding to a difference of $\Delta n_{\mathrm{eff}}=0.018$ in effective index for the guided and leaky mode.

\section{SUMMARY}

NSOM was used for the first time to directly image transient interference between the guided mode and a leaky mode induced in a single-mode waveguide due to a localized core thick-

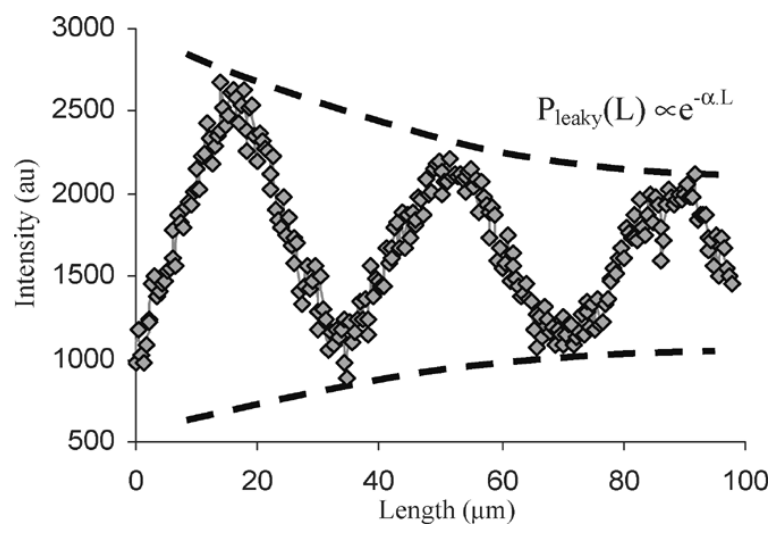

Fig. 6. Measured optical intensity oscillation after adlayer (diamonds) and exponential decay fit to envelope amplitude (dashed line).

ness change. The attenuation and relative propagation constant of the leaky mode can be extracted using this method. These values, as well as the field perturbation within the adlayer and general optical profile, were in good agreement with BPM simulations. The transient interference following an individual adlayer region in an LEAC sensor may limit the proximity of separate adlayer regions, but may be decreased by engineering the waveguide to minimize the excitation of leaky modes or by rapidly attenuating these modes. The strength of the oscillation may be a useful indication of the adlayer's optical properties.

\section{ACKNOWLEDGMENT}

The authors would like to thank Dr. Van Orden for access to NSOM, and Thermo-Electron, Inc. for $\operatorname{SiN}_{x}$ film deposition

\section{REFERENCES}

[1] G. W. Yuan, M. D. Stephens, D. S. Dandy, and K. L. Lear, "Novel local evanescent field detection waveguide multianalyte biosensor," in SPIE Proc., vol. 5557, Oct. 2004, pp. 140-146.

[2] G. H. Vander Rhodes, B. B. Goldberg, M. S. Ünlü, S. T. Chu, W. Pan, T. Kaneko, Y. Kokobun, and B. E. Little, "Measurement of internal spatial modes and local propagation properties in optical waveguides," Appl. Phys. Lett., vol. 75, no. 16, pp. 2368-2370, Oct. 1999.

[3] S. Bourzeix, J. M. Moison, F. Mignard, F. Barthe, A. C. Borccara, C. Licoppe, B. Mersali, M. Allovon, and A. Bruno, "Near-field optical imaging of light propagation in semiconductor waveguide structures," Appl. Phys. Lett., vol. 73, no. 8, pp. 1035-1037, 1998.

[4] W. Ji, D. Kim, H. J. Kim, O. Beom-Hoan, S. Park, E. Lee, and S. G. Lee, "NSOM-based characterization method applicable to optical channel waveguide with a solid-state cladding," IEEE Photon. Technol. Lett., vol. 17, no. 4, pp. 846-848, Apr. 2005.

[5] A. L. Campillo, J. W. P. Hsu, K. R. Parameswaran, and M. M. Fejer, "Direct imaging of multimode interference in a channel waveguide," Opt. Lett., vol. 28, no. 6, pp. 399-401, Mar. 2003.

[6] N. F. Van Hulst, M. H. P. Moers, O. F. J. Noordman, R. G. Tack, and F. B. Segerink, "Near-field optical microscope using a silicon-nitride probe," Appl. Phys. Lett., vol. 62, no. 5, pp. 461-463, Feb. 1993.

[7] P. Clauberg and P. Von Allmen, "Vectorial beam-propagation method for integrated optics,” Electron. Lett., vol. 27, pp. 654-655, 1991. 